\title{
OUTPUT ERROR IDENTIFICATION IS NOT GLOBALLY STABLE IN THE "NONIDEAL" CASE
}

William A. Sethares and Dale A. Lawrence

Dept. of Electrical \& Computer Engineering University of Wisconsin Madison, WI 53706

Abstract

This rote demonstrates that the parameter estimates of the output error identification algorithm are not necessarily globally bounded when the prediction error is corrupted by an arbitrarily small disturbance. The cause of the parameter estimate drift to infinity is an input which can be interpreted as an open loop control to an unstable system. The disturbance may actually be vanishing.

\section{Introduction}

This note presents several scalar examples of the misapplication of the output error identification scheme [1] in nonideal situations. Global stability of the algorithm (which is true under assumptions of persistence of excitation (PE) and strict positive reality (SPR) [2] of the denominator of the unknown pant) is shown to fail when these assumptions are relaxed. This failure is demonstrated by constructing examples where the parameter estimates diverge to infinity. The examples are constructed for the scalar (and hence SPR) case where the model is of the exact same linear structure as the unknown plant, but where the measurement of the prediction error is corrupted by an order $\varepsilon$ disturbance. A bounded input sequence, combined with the order $\varepsilon$ disturbance, slowly drives the parameter estimates to infinity.

This possibility of drifting to infinity may be surprising. Indeed, a folklore has grown up concerning the global behavior of the output error identifier. Suppose the SPR condition holds and that some unfortunate set of circumstances (lack of PE, disturbances) arise in which the parameter estimates wander outside the unit circle (if they never leave the unit circle, then they're clearly bounded). The model would then be unstable, and would cause a large prediction error, in turn creating excitation. This self generated excitation would then cause a restabilization (since the algorithm is globally bounded with PE and SPR). We show that this folkloric restabilization need not always occur.

When the SPR condition fails, it is possible to find inputs (or excitation sequences) that cause the equilibrium to be locally unstable [3], [4]. Simulations indicate that the parameter estimate trajectories approach this equilibrium, are repelled, move outside the stability region, and then restabilize. The assumed explanation is, once again, that the exponential growth of the prediction error causes adequate excitation in frequency regions where the plant is SPR, thus causing a restabilization that is again due to self generated excitation.

How, then, could global stability fail? Something must "cancel out" the self generated excitation which threatens to restabilize the algorithm.

One possibility is to suppose that two poles leave the unit circle simultaneously [5]. While the algorithm struggles to restabilize one pole, the other might continue growing. While the algorithm struggled to stabilize the other, the first one might grow. The two might then alternately bounce towards infinity.

A simpler idea, and the one which will be pursued here, is to suppose that something literally
Martin Marrietta Corporation Boulder, CO 80201

cancels out the "exponential growth" before it has a chance to grow. The strategy in constructing these examples is to approach the problem backwards... consider the following sequence of questions: How might the parameter estimate grow? What prediction error would allow such growth? What estimated output would allow such a prediction error? What input sequence would cause such an estimated output?

Our contention is not that such unbounded behavior is likely in a realistic problem setting. Indeed, the examples are quite singular in the sense that inputs arbitrarily close to those considered will often lead to bounded, rather than unbounded, parameter estimates. Rather, these examples show definitively that the output error algorithm in the nonideal setting (and without PE) is not globally bounded. Moreover, we are able to describe the set of situations which lead to such parameter drift as essentially "open loop controls to unstable plants." Such insights lead towards a deeper understanding of the interplay between the local and global stability characteristics of adaptive algorithms.

\section{Problem Setup}

A stable first order plant with unknown pole location $|a|<1$ is to be estimated by the output error scheme. The plant output is

$$
y_{k+1}=a y_{k}+u_{k}
$$

where $u_{k}$ is the input, and the estimated output is

$$
\hat{y}_{k+1}=\hat{a}_{k+1} \hat{y}_{k}+u_{k} \text {. }
$$

Suppose that the prediction error $y_{k}-\hat{y}_{k}$ can only be measured in the presence of a disturbance $w_{k}$. Then

$$
e_{k+1}=y_{k+1}-\hat{y}_{k+1}+w_{k+1} \text {, }
$$

and the parameter estimate update is

$$
\hat{a}_{k+1}=\hat{a}_{k}+\mu \hat{y}_{k} e_{k+1}
$$

where $\mu$ is a small positive stepsize. Substituting (1) and (2) into (3) and then into (4) gives

$$
\hat{a}_{k+1}=\left(1-\mu \hat{y}_{k}^{2}\right) \hat{a}_{k}+\mu \hat{y}_{k}\left(a y_{k}+w_{k}\right) \text {, }
$$

which describes the time evolution of the parameter estimate $\hat{a}_{\mathbf{k}}$. The strategy of the succeeding examples is to examine the parameter update equation (5), and find examples where particular choices of bounded $u_{k}$ and $w_{k}$ cause $\hat{a}_{k}$ to become unbounded. Note that (2) is an unnormalized a posteriori update, which is equivalent to a normalized a priori update.

\section{The Examples}

The form of equation (5) is revealing. Notice that if $\hat{y}_{k}$ were persistently exciting [6], then the system (5) would be exponentially asymptotically stable, and hence would be bounded-input bounded-output stable. In particular, bounded $w_{k}$ would imply bounded $\hat{a}_{k}$. To construct an example of unbounded drift, then, $\hat{y}_{k}$ must fail to be PE. One way to fail the $P E$ condition is to have $\hat{y}_{k}$ converge to zero. This idea is exploited repeatedly in the following examples. 
EXAMPLE 1: Let $u_{k}=\frac{1}{k+1}-\frac{\mu \varepsilon}{k} \sum_{i=1}^{k} \frac{1}{i}$. Note that $u_{k} \rightarrow 0$ as $k \rightarrow \infty$ since $\sum_{i=1}^{k} \frac{1}{i}$ is approximately $\ln k$, and $\frac{\ln k}{k} \rightarrow 0$ as $k \rightarrow \infty$. From (1), $y_{k} \rightarrow 0$. Let the disturbance $w_{k}$ be defined such that $e_{k}=\varepsilon$ for every $k$ Then, assuming the initial conditions $\hat{y}_{1}=1$ and $\hat{\mathrm{a}}_{1}=\mu \varepsilon$, the solution to (2) and (4) is $\hat{\mathrm{y}}_{\mathrm{k}}=\frac{1}{\mathrm{k}}$ and $a_{k+1}=\mu \varepsilon \sum_{j=1}^{k} \frac{1}{i}$, which is easily verified by direct substitution. Note that $w_{k}$ is chosen so that $w_{k+1}=\varepsilon-y_{k+1}+\frac{1}{k+1}$, which is clearly bounded. In fact, $w_{k}$ converges to $\varepsilon$ as $k \rightarrow \infty . \Delta \Delta \Delta$

This shows that the output error identifier is not globally bounded in the presence of bounded measure ment disturbances, since â diverges to infinity. The parameter drift of example 1 arises because the excitation vanishes. At first glance, it might seem necessary that the input $u_{k} \rightarrow 0$ in order to drive $\hat{y}$ to zero. This is not so. Example 2 shows that $u_{k}$ can be bounded away from zero, and still drive the parameter estimate

to infinity. choose $w_{k}$ so that $e_{k}=\varepsilon$ for every $k$. With initial conditions as in example 1 , the solution to (2) and (4) is now $\hat{a}_{k+1}=\mu \varepsilon \sum_{i=1}^{k} \frac{1}{\sqrt{i}}$ and $\hat{y}_{k}=\frac{1}{\sqrt{\bar{k}}}$, which may again be verified by direct substitution. To see that $u_{k}$ is bounded away from zero, note that

$$
\begin{aligned}
\lim _{\mathrm{k} \rightarrow \infty} u_{k} & \leq-\mu \varepsilon \lim _{\mathbf{k} \rightarrow \infty} \frac{1}{\sqrt{\mathrm{k}}} \int_{\mathrm{i}=1}^{\mathrm{k}} \frac{\mathrm{di}}{\sqrt{\mathrm{i}}} \\
& \leq-\mu \varepsilon \lim _{\mathrm{k} \rightarrow \infty} \frac{2 \sqrt{\mathrm{k}}-2}{\sqrt{\bar{k}}}=-2 \mu \varepsilon .
\end{aligned}
$$

Since (1) is stable, $y_{k}$ is bounded, and $w_{k+1}=\varepsilon-y_{k+1}+\frac{1}{\sqrt{k+1}}$ converges to a constant

related to $\varepsilon$ and the d.c. gain of the plant. $\Delta \Delta \Delta$

As $\hat{a}_{k}$ drifts to larger values, the recursion (2) becomes more unstable. Suppose that $\hat{a}$ is driven towards infinity. The output can remain bounded only if $\hat{y}_{\mathbf{k}}$ converges to zero. This means that $\hat{a}_{k+1} \hat{y}_{k}+u_{k} \rightarrow 0$, which in turn implies that $u_{k} \rightarrow-\hat{a}_{k+1} \hat{y}_{k}$. This is formalized as

LEMMA: Suppose $\hat{a}_{k} \rightarrow \infty$ and yet the output of the recursion (2) remains bounded. If $\mathrm{u}_{\mathrm{k}}$ is bounded, then $u_{k}$ must converge to $-\hat{a}_{k+1} \hat{y}_{k}$.

PROOF: Suppose the bounds on $y_{k}$ and $u_{k}$ are given by $\|\hat{\mathbf{y}}\|_{\infty}$ and $\|\mathrm{u}\|_{\infty}$. First, we show that $\hat{\mathrm{y}}$ must converge to zero. Suppose not. Then there exists an $\varepsilon>0$ such that $\left|\hat{y}_{k}\right|>\varepsilon$ for infinitely many $k$. Choose $k$ large enough so that $\left|\hat{a}_{\mathbf{k}+1}\right|>\frac{\|\hat{\mathbf{y}}\|_{\infty}+\prod \mathbf{u} \|_{\infty}}{\varepsilon}$. Then $\left|\hat{y}_{k+1}\right|>\varepsilon\left\{\frac{\|\hat{y}\|_{\infty}^{\varepsilon}+\|\mathbf{u}\|_{\infty}}{\varepsilon}\right\}-\|u\|_{\infty}>\|\hat{y}\|_{\infty}$, contradicting the bound on $\hat{\mathrm{y}}$. Thus $\hat{\mathrm{a}}_{\mathrm{k}+1} \hat{\mathrm{y}}_{\mathrm{k}}+\mathrm{u}_{\mathrm{k}} \rightarrow 0$. $\Delta \Delta \Delta$

This sequence $u_{k}$ can be thought of as a "control" for an unstable system since the nature of the input masks the instability of the system. Note that $u_{k}$ could be constructed by feedback of the signals $\hat{a}_{k+1}$ and $\hat{y}_{k}\left(\hat{a}_{k+1}\right.$ can be constructed as a function of $\hat{a}_{k}$ and $\hat{y}_{k}$ ). Since there is no feedback loop in the output error identifier, situations in which this sort of drift will arise are limited to inputs which are, essentially, open loop controls to unstable plants. This suggests, however, that when the identifier is embedded in a feedback loop (such as in the adaptive control problem), care must be taken to avoid such "control" sequences.

It may appear that the biased persistence of $w_{k}$ is necessary for such drift to occur. In fact, such drift can occur even if the disturbance converges to zero.

EXAMPLE 3: Let $\mathrm{u}_{\mathrm{k}}=\frac{1}{(\mathrm{k}+1)^{\mathrm{b}}}-\frac{\mu \varepsilon}{\mathrm{k}^{\mathrm{b}}} \sum_{\mathrm{i}=1}^{\mathrm{k}} \frac{1}{\mathrm{i}^{\mathrm{a}+\mathrm{b}}}$, and suppose that $e_{k+1}=\frac{1}{k^{a}}$. Then $\hat{y}_{k}=\frac{1}{k^{b}}$ and $\hat{a}_{k+1}=\mu \sum_{i=1}^{k} \frac{1}{i^{a+b}}$. If $a$ and $b$ are such that

(1) $a+b \leq 1$, and

(2) $a+2 b>1$,

then $\hat{a}_{k}$ diverges to infinity.

PROOF: (1) implies the divergence of $\hat{a}_{k}$. (2) ensures that $u_{k}$ converges to zero, which implies that $y_{k} \rightarrow 0$, and hence that $w_{k+1}=\frac{1}{(k+1)^{a}}-y_{k+1}+\frac{1}{(k+1)^{b}}$ converges to zero. $\Delta \Delta \Delta$

\section{Conclusions}

Several interesting questions are raised by these examples of parameter drift in the output error algorithm in nonideal usage:

(1) Can similar examples be constructed to demonstrate instability of the output error algorithm when there is no measurement noise, but instead a small nonideality is introduced in the plant?

(2) One may think of the input-disturbance pairs that cause divergence in these examples as a "trajectory" or "manifold" in state space. Can this be proven unstable for any input disturbance pair causing divergence? The classification of such pairs as open loop controls to unstable plants gives a conceptual tool with which to attack this problem.

(3) Can these examples be extended to non-SPR plants?

(4) The finding of sufficient conditions for divergence of an algorithm is equivalent to finding necessary conditions for boundedness. Examples such as these may help to unravel a useful set of necessary and sufficient conditions for proper behavior of algorithms such as the output error identifier.

\section{References}

[1] Johnson, C. R. Jr., "Adaptive IIR filtering: current results and open issues," IEEE Trans. on Information Theory, vol. IT-30, no. 2, pp. 237-250, March 1984.

[2] Bitmead R. R., "Persistence of excitation conditions and the convergence of adaptive schemes, "IEEE Trans. on Information Theory, vol. IT-30, no. 2, pp. 183-191, March 1984.

[3] Schoenwald D., P. Kokotovic, and S. Dasgupta, "A boundedness conjecture for locally unstable estimation of non-SPR transfer functions," Proc. 21st Conference on Information and Systems Sciences, Johns Hopkins, Baltimore, MD. pp. 318-322, March 1987.

[4] Anderson B. D. O., R. R. Bitmead, C. R. Johnson, Jr., P. V. Kokotovic, R. L. Kosut, I. M. Y. Mareels, L. Praly, B. D. Reidle, Stability of Adaptive Systems: Passivity and Averaging Analysis, MIT Press, 1986.

[5] private correspondence J. Moore, Australian National University, Canberra, ACT, Australia. 